\title{
A TOPOLOGICALLY STRONGLY MIXING SYMBOLIC MINIMAL SET
}

\author{
BY \\ K. E. PETERSEN $\left({ }^{1}\right),\left({ }^{2}\right)$
}

\begin{abstract}
Recent papers by the author, Keynes and Robertson, and others have shown that weakly mixing minimal flows are objects of considerable interest, but examples of such flows, other than the horocycle flows, have been scarce. We give here a "machinal" construction of a bilateral sequence with entries from $\{0,1\}$ whose orbit closure is topologically strongly mixing and minimal. We prove in addition that the flow we obtain has entropy zero, is uniquely ergodic, and fails to be measuretheoretically strongly mixing.
\end{abstract}

1. Mixing for symbolic flows. In this section we give some definitions and establish criteria for the weak and strong mixing of symbolic flows. For unexplained notation and terminology the reader may consult [2], [3], [4], and [7].

Let $X$ be a compact metric space and $\phi: X \rightarrow X$ a homeomorphism; the pair $(X, \phi)$ is called a flow. If $(X, \phi)$ is a flow and $x \in X$, the orbit of $x$ is $\mathcal{O}(x)=$ $\left\{\phi^{n} x: n \in Z\right\}$, where $Z$ denotes the set of integers. A flow $(X, \phi)$ is said to be minimal if for each $x \in X$ we have $\mathcal{O}(x)^{-}=X$, ergodic if there is $x \in X$ such that $\mathcal{O}(x)^{-}=X$, weakly mixing if the product flow $(X \times X, \phi)$ is ergodic, and strongly mixing if given nonempty open subsets $A$ and $B$ of $X$ there is $n_{0} \in Z$ such that $\phi^{n} A \cap B \neq \varnothing$ whenever $|n| \geqq n_{0}$. Observe that a flow $(X, \phi)$ is ergodic if and only if given nonempty open subsets $A$ and $B$ of $X$ there is $n \in Z$ such that $T^{n} A \cap B \neq \varnothing$. It can be proved [12] that $(X, \phi)$ is weakly mixing if and only if given nonempty open subsets $A$ and $B$ of $X$ there is $n \in Z$ such that $\phi^{n} A \cap A \neq \varnothing$ and $\phi^{n} A \cap B \neq \varnothing$.

Let $\{0,1\}$ have the discrete topology and let

$$
S=\prod_{-\infty}^{\infty}\{0,1\}=\{0,1\}^{z}
$$

so that $S$ is a compact metric space. We will think of elements $x \in S$ as being bilateral sequences of 0 's and 1's, $x=\cdots x_{-2} x_{-1} \dot{x}_{0} x_{1} x_{2} \ldots$, with the dot marking

Presented to the Society, January 23, 1969; received by the editors July 11, 1969.

AMS Subject Classifications. Primary 5470; Secondary 2833.

Key Words and Phrases. Minimal sets, strong mixing, weak mixing, symbolic dynamics.

(1) Research supported by Army Research Office (Durham).

$\left({ }^{2}\right)$ The contents of this paper were previously announced in [11] and are contained in the author's doctoral dissertation prepared at Yale University under the direction of Professor S. Kakutani. The author wishes to express his gratitude to Professor Kakutani, Professor G. A. Hedlund, and Professor M. Keane for their assistance and encouragement in the preparation of this research. 
the "central position" of the sequence $x$. A metric compatible with the topology of $S$ is given by, for example, $d(x, y)=1 /(1+k)$, where

$$
k=\inf \left\{|n|: x_{n} \neq y_{n}\right\}, \quad x, y \in S .
$$

If $k$ is a positive integer, a $k$-block $B$ will be an ordered $k$-tuple $B=b_{1} b_{2} \cdots b_{k}$, where each $b_{i}$ is 0 or 1 for $i=1,2, \ldots, k$. We will say that $C$ is a block in case $C$ is a $k$-block for some $k$. If $x \in S, B=b_{1} b_{2} \cdots b_{k}$ is a $k$-block, and $n \in Z$ is such that $x_{n}=b_{1}, x_{n+1}=b_{2}, \ldots, x_{n+k-1}=b_{k}$, then we will say that $B$ appears at the $n$th place in $x$. The phrase $B$ appears in $x$ will mean that there is $n \in Z$ such that $B$ appears at the $n$th place in $x$. If $k$ is a nonnegative integer and $A$ is a $(2 k+1)$-block which appears at the $(-k)$ th place in a sequence $x \in S$, then $A$ will be called the central $(2 k+1)$-block of $x$. A block $D$ will be called a central block of a sequence $x \in S$ if there is a $k$ such that $D$ is the central $(2 k+1)$-block of $x$. It is not difficult to see that if $x \in S$ then the family $\{\{y \in S: A$ is a central block of $y\}: A$ is a central block of $x$ \} forms a neighborhood base at $x$.

The shift transformation $\sigma: S \rightarrow S$ is defined by $(\sigma x)_{n}=x_{n+1}$ for all $x \in S$. The map $\sigma$ is a homeomorphism of $S$ onto $S$, and thus the pair $(S, \sigma)$ is a flow. If $R$ is a closed subset of $S$ such that $\sigma R=R$, then the pair $(R, \sigma \mid R)$, usually written just $(R, \sigma)$, is called a symbolic flow.

If $B$ is a $k$-block, the length of $B$ will be defined to be $L(B)=k$. Let $q$ and $p$ be positive integers with $q \leqq p$, let $E=e_{1} e_{2} \cdots e_{q}$ be a $q$-block, $F=f_{1} f_{2} \cdots f_{p}$ a $p$-block, and let $j \in Z$ be such that $1 \leqq j \leqq p-q+1$. We will say that $E$ appears at the jth place in $F$ if $f_{j}=e_{1}, f_{j+1}=e_{2}, \ldots, f_{j+q-1}=e_{q}$. If $E$ and $F$ are blocks, we will say that $E$ appears in $F$ in case there is a $j$ such that $E$ appears at the $j$ th place in $F$.

Let $x \in S$ and let $X=\mathcal{O}(x)^{-}$. For each block $C$ which appears in $x$, let $\mathscr{N}(C)$ $=\{n: C$ appears at the $n$th place in $x\}$. If $C$ and $D$ are blocks which appear in $x$, we define $\mathscr{N}(C, D)=\{m-n: m \in \mathscr{N}(C), n \in \mathscr{N}(D)\}$. The following theorems are stated without proof, since they are little more than direct translations of the definitions of weak and strong mixing into the framework of symbolic dynamics.

THEOREM 1.1. Let $x \in S$ and let $X=\mathcal{O}(x)^{-}$. Then the following statements are equivalent:

(1) $(X, \sigma)$ is weakly mixing.

(2) For any two blocks $A$ and $B$ which appear in $x$ we have

$$
\mathscr{N}(A, A) \cap \mathscr{N}(B, A) \neq \varnothing .
$$

(3) For each central block $A$ of $x$ and each $L(A)$-block $B$ which appears in $x$ we have

$$
\mathscr{N}(A, A) \cap \mathscr{N}(B, A) \neq \varnothing .
$$

TheOREM 1.2. Let $x \in S$ and let $X=\mathcal{O}(x)^{-}$. Then the following statements are equivalent:

(1) $(X, \sigma)$ is strongly mixing. 
(2) For any two blocks $A$ and $B$ which appear in $x$ there is an integer $n_{0}$ such that

$$
\mathscr{N}(A, B) \supseteq\left\{n \in Z:|n| \geqq n_{0}\right\} \text {. }
$$

(3) For each central block $A$ of $x$ there is an integer $n_{0}$ such that

$$
\mathscr{N}(A, A) \supseteq\left\{n \in Z:|n| \geqq n_{0}\right\} \text {. }
$$

(4) There is a sequence $\left\{A_{k}: k=0,1,2, \ldots\right\}$ of central blocks of $x$ such that $L\left(A_{k}\right) \rightarrow \infty$ and such that for each $k=0,1,2, \ldots$ there is an integer $n_{0}(k)$ such that

$$
\mathscr{N}\left(A_{k}, A_{k}\right) \supseteq\left\{n \in Z:|n| \geqq n_{0}(k)\right\} .
$$

2. Construction of $(W, \sigma)$. For any block $A=a_{1} a_{2} \cdots a_{p}$ and for $1 \leqq i \leqq p$ we define $A[i]=a_{i} a_{i+1} \cdots a_{p} a_{1} a_{2} \cdots a_{p-1}$, the cyclic permutation of $A$ which has its initial point at the $i$ th place of $A$.

We construct a sequence of blocks $A_{k}$ in the following manner. Given $A_{i}$ for $i \leqq k, A_{k+1}$ is obtained from $A_{k}$ by writing down in succession certain "allowable" cyclic permutations $A_{k}[i]$ of $A_{k}$ and alternating these with repetitions of $A_{k}$ itself. The "allowable" cyclic permutations of $A_{k}$ are those which split no appearance (which is explicit in the construction) of $A_{i}$ in $A_{k}$, for $i<k$. Forbidding certain cyclic permutations of $A_{k}$ allows us to be certain that the sequence so defined is almost periodic; and there are still enough "allowable" cyclic permutations of $A_{k}$ available to assure us that the sequence has a weakly mixing orbit closure.

In the following the $A_{k}$ will be a sequence of central blocks of the element $w \in S$ to be defined, and $\mathfrak{A}(k)$ will denote the set of places in $A_{k}$ (counted from the left endpoint of $A_{k}$ ) which are allowable as initial points of cyclic permutations of $A_{k}$. The $B_{k}$ will be an auxiliary sequence of blocks used to determine the $\mathfrak{A}(k)$.

Specifically, then, define $A_{0}=101, \mathfrak{A}(0)=\{1,2,3\}$, and $B_{0}=111$. For each $k$, $m_{k}$ will denote the cardinal of the set $\mathfrak{A}(k), L_{k}$ will denote $L\left(A_{k}\right)=L\left(B_{k}\right)$, and $E_{k}$ will denote the $L_{k}$-block all of whose entries are 0 except the first, which is 1 .

Assume now that $A_{k}, B_{k}$, and $\mathfrak{r}(k)=\left\{i_{1}, i_{2}, \ldots, i_{m_{k}}\right\}$ with $1=i_{1}<i_{2}<\cdots<i_{m_{k}}$ have been defined. Then we define

and

$$
A_{k+1}=A_{k} A_{k}\left[i_{m_{k}}\right] A_{k} \cdots A_{k} A_{k}\left[i_{2}\right] A_{k} A_{k}\left[i_{2}\right] A_{k} \cdots A_{k} A_{k}\left[i_{m_{k}}\right] A_{k}
$$

$$
B_{k+1}=E_{k} B_{k}\left[i_{m_{k}}\right] E_{k} \cdots E_{k} B_{k}\left[i_{2}\right] E_{k} B_{k}\left[i_{2}\right] E_{k} \cdots E_{k} B_{k}\left[i_{m_{k}}\right] E_{k} .
$$

If $B_{k+1}=b_{1} b_{2} \cdots b_{L_{k+1}}$, we then define $\mathfrak{2}(k+1)=\left\{j: 1 \leqq j \leqq L_{k+1}\right.$ and $\left.b_{j}=1\right\}$.

Thus $B_{k+1}$ has the function of marking the places in $A_{k+1}$ which are allowable as initial points of cyclic permutations of $A_{k+1}$ : if 1 appears at the $j$ th place in $B_{k+1}$, then $j \in \mathfrak{A}(k+1)$ and $A_{k+1}[j]$ appears in $A_{k+2}$. We notice also that if $1 \leqq j \leqq L_{k+1}$ and $j \equiv 1\left(\bmod L_{k}\right)$, then $j \in \mathfrak{A}(k+1)$.

By way of illustration,

$$
\begin{aligned}
A_{1} & =101110101011101011101110101, \\
B_{1} & =100111100111100111100111100, \text { and } \\
\mathfrak{R}(1) & =\{1,4,5,6,7,10,11,12,13,16,17,18,19,22,23,24,25\} .
\end{aligned}
$$


In this way, then, $A_{k}, B_{k}$, and $\mathfrak{A}(k)$ are defined by induction for all integers $k \geqq 0$. For each $k \geqq 0$ let $w_{k}$ be that element of $S$ whose central $L_{k}$-block is $A_{k}$ and all of whose other entries are zero. Since $L_{k} \rightarrow \infty$ and the central $L_{k}$-subblock of $A_{k+1}$ is $A_{k}, \lim _{k} w_{k}=w$ exists. We put $W=\mathcal{O}(w)^{-}$.

From the definition of $B_{k+1}$ we observe that $m_{k+1}=2 m_{k}^{2}-1$ and $L_{k+1}=$ $\left(4 m_{k}-3\right) L_{k}$. Each $m_{n}$ is in fact a $\left(2^{n+1}\right)$ th solution of the Pell equation $x^{2}-2 y^{2}=1$. For $(1-\sqrt{ } 2)(1+\sqrt{ } 2)=-1$, so for $n \geqq 1$ we have $(1-\sqrt{ } 2)^{2^{n}}(1+\sqrt{ } 2)^{2^{n}}=1$. For each $n \geqq 1$ write $(1-\sqrt{ } 2)^{2^{n}}=x_{n}-\sqrt{ } 2 y_{n}$, with $x_{n}, y_{n} \in Z$. Then $(1+\sqrt{ } 2)^{2^{n}}=$ $x_{n}+\sqrt{ } 2 y_{n}$, so $1=\left(x_{n}-\sqrt{ } 2 y_{n}\right)\left(x_{n}+\sqrt{ } 2 y_{n}\right)=x_{n}^{2}-2 y_{n}^{2}$. However,

$$
(1-\sqrt{ } 2)^{2^{n}}=\left(x_{n-1}-\sqrt{ } 2 y_{n-1}\right)^{2}
$$

so we have the relation $x_{n}=x_{n-1}^{2}+2 y_{n-1}^{2}=2 x_{n-1}^{2}-1$. Since $x_{1}=3=m_{0}$, we may conclude that $m_{n}=x_{n+1}$ for all integers $n \geqq 0$.

3. Strong mixing, minimality, and topological entropy. We prove in this section that the flow $(W, \sigma)$ is strongly mixing and minimal and has topological entropy zero. We need to know first that our definition of the sequence $w$ employs "enough" cyclic permutations at each stage of the construction.

For each $k=0,1,2, \ldots$ let $\mathfrak{B}(k)=\left\{j: 1 \leqq j \leqq L_{k}\right.$ and there is $i \in \mathfrak{A}(k)-\{1\}$ such that 1 appears at the $j$ th place in $\left.B_{k}[i]\right\}$. Then if $r \in \mathfrak{B}(k)$, we may split $A_{k+1}$ at the $r$ th place of $A_{k}[i]$, for some $i \in \mathfrak{A}(k)$, in order to form a permutation $A_{k+1}[j]$ which appears in $A_{k+2}$.

LEMMA 3.1. For each $k=0,1,2, \ldots, \mathfrak{B}(k) \supseteq\left\{n \in Z: 1 \leqq n \leqq L_{k}\right\}$.

Proof. We proceed by induction. For $k=0, B_{0}=B_{0}[2]=B_{0}[3]=111$, so clearly $\mathfrak{B}(0)=\{1,2,3\} \supseteq\left\{n \in Z: 1 \leqq n \leqq L_{0}\right\}$. Suppose now that $k \geqq 0$ and

$$
\mathfrak{B}(k) \supseteq\left\{n \in Z: 1 \leqq n \leqq L_{k}\right\},
$$

and let $n \in Z$ with $1 \leqq n \leqq L_{k+1}$ be given. We need to find $i \in \mathfrak{A}(k+1)-\{1\}$ such that 1 appears at the $n$th place in $B_{k+1}[i]$.

Let $n^{\prime} \equiv n\left(\bmod L_{k}\right)$ and $1 \leqq n^{\prime} \leqq L_{k}$; suppose $n=n^{\prime}+p L_{k}$. By induction there is $j \in \mathfrak{A}(k)-\{1\}$ such that 1 appears at the $\left(n^{\prime}\right)$ th place in $B_{k}[j]$. Now $B_{k}[j]$ appears in $B_{k+1}$, since $j \neq 1$. Because $r \equiv 1\left(\bmod L_{k}\right)$ implies $r \in \mathfrak{A}(k+1)$, and because $B_{k}[j]$ appears at least twice in $B_{k+1}$, there is $i \in \mathfrak{A}(k+1)-\{1\}$ such that $B_{k}[j]$ appears at the $\left(p L_{k}+1\right)$ th place in $B_{k+1}[i]$. But then we see that 1 appears at the $n$th place in $B_{k+1}[i]$.

THEOREM 3.1. $(W, \sigma)$ is strongly mixing.

Proof. Using Theorem 1.2, it suffices to prove that for each $k=0,1,2, \ldots$ there is an integer $n_{0}(k)$ such that $\mathscr{N}\left(A_{k}, A_{k}\right) \supseteq\left\{n \in Z:|n| \geqq n_{0}(k)\right\}$. (Here the $A_{k}$ 's are as in §2.)

Given $k=0,1,2, \ldots$, let $n_{0}(k)=L_{k}$ and let $N \in Z$ with $|N| \geqq n_{0}(k)$ be given. Now the block $A_{k} A_{k}$ appears in $A_{k+2}$, so if $|N|=n_{0}(k)$, then $N \in \mathscr{N}\left(A_{k}, A_{k}\right)$; 
thus we may assume that $|N| \geqq n_{0}(k)+1$. Choose $m$ such that $L_{m} \geqq|N|$, so $L_{m}>L_{k}$ and $A_{k}$ appears in $A_{m}$. If $r=L_{m}-|N|+L_{k}+1$, then $1 \leqq r \leqq L_{m}$, so by the lemma there is $j \in \mathfrak{A}(m)-\{1\}$ such that 1 appears at the $r$ th place in $B_{m}[j]$. If $A_{m}[j]=$ $q_{1} q_{2} \cdots q_{L_{m}}$, then there is $i \in \mathfrak{A}(m+1)$ such that

$$
A_{m+1}[i]=q_{r} q_{r+1} \cdots q_{L_{m}} A_{m} \cdots A_{m} q_{1} q_{2} \cdots q_{r-1} .
$$

But then the block $A_{m+1} A_{m+1}[i]$ appears in $w$ and contains the block $A_{k} q_{r} q_{r+1} \ldots$ $q_{L_{m}} A_{k}$, so we see that $L_{k}+L_{m}-r+1 \in \mathcal{N}\left(A_{k}, A_{k}\right)$. Since $L_{k}+L_{m}-r+1=|N|$, we have $N \in \mathscr{N}\left(A_{k}, A_{k}\right)$.

In order to prove that the sequence $w$ is almost periodic, we need to know that the appearances of $A_{i}$ which are explicit in the construction are not split as we proceed with the construction of $w$. To this end we introduce another auxiliary sequence of blocks $\Lambda_{k}$, with entries from $Z$. Define $\Lambda_{0}=(-1)(-1)(-1)$ and $\Lambda_{0}^{\prime}=(0)(-1)(-1)$, where the parentheses serve only to separate the entries in a block from one another. If $k$ is a nonnegative integer and $\Lambda_{k}$ and $\Lambda_{k}^{\prime}$ have been defined, define

$$
\Lambda_{k+1}=\Lambda_{k}^{\prime} \Lambda_{k}\left[i_{m_{k}}\right] \Lambda_{k}^{\prime} \cdots \Lambda_{k}^{\prime} \Lambda_{k}\left[i_{2}\right] \Lambda_{k}^{\prime} \Lambda_{k}\left[i_{2}\right] \Lambda_{k}^{\prime} \cdots \Lambda_{k}^{\prime} \Lambda_{k}\left[i_{m_{k}}\right] \Lambda_{k}^{\prime},
$$

where the $i_{j}$ 's are as in $\S 2$. If $\Lambda_{k+1}=\lambda_{1} \lambda_{2} \cdots \lambda_{s}$ with each $\lambda_{i} \in Z$, define $\Lambda_{k+1}^{\prime}$ $=(k+1) \lambda_{2} \lambda_{3} \cdots \lambda_{s}$. Thus $\Lambda_{k}$ and $\Lambda_{k}^{\prime}$ are defined by induction for all integers $k \geqq 0$. Note that $L\left(\Lambda_{k}\right)=L\left(\Lambda_{k}^{\prime}\right)=L_{k}$ for all $k$.

Let $m$ and $k$ be integers with $m \geqq k \geqq 0$ and suppose that $\Lambda_{m}^{\prime}=\lambda_{1} \lambda_{2} \cdots \lambda_{s}$ with each $\lambda_{i} \in Z$. The following statements are established by straightforward induction arguments.

(1) If $\lambda_{j} \geqq k$, then the $L_{k}$-subblock of $B_{m}$ appearing at the $j$ th place in $B_{m}$ is either $000 \cdots 00$ or $100 \cdots 00$.

(2) If $\lambda_{j} \geqq k$, then the $L_{k}$-subblock of $A_{m}$ appearing at the $j$ th place in $A_{m}$ is $A_{k}$.

(3) If $1 \leqq i \leqq j \leqq L_{m}, \lambda_{i} \geqq k$ and $\lambda_{j} \geqq k$, and if $i \leqq p \leqq j$ implies $\lambda_{p}<k$, then $j-i \leqq L_{k}$.

These three statements imply that we may write $A_{m}=A_{k} C_{1} A_{k} C_{2} A_{k} \cdots A_{k} C_{r} A_{k}$, where $L\left(C_{j}\right) \leqq L_{k}$ for $j=1,2, \ldots, r$. Therefore $A_{k}$ appears in each $A_{m}$, and hence in $w$, with bounded gap. It follows that the sequence $w$ is almost periodic, and so we may state the following theorem.

- Theorem 3.2. $(W, \sigma)$ is minimal.

For each $n=1,2, \ldots$ let $\theta(n)$ denote the number of different $n$-blocks which appear in the sequence $w$. Then the limit

$$
K(W)=\lim _{n \rightarrow \infty} \frac{\log \theta(n)}{n}
$$

exists and is the topological entropy (see [1], [2], and [4]) of the flow $(W, \sigma)$. We wish to prove that $K(W)=0$. For this purpose it suffices to prove that

$$
\lim _{k \rightarrow \infty} \frac{\log \theta\left(L_{k}\right)}{L_{k}}=0 .
$$


LEMMA 3.2. If $k$ is a nonnegative integer and $B$ is an $L_{k}$-block which appears in $w$, then $B$ appears in $A_{k+2}$.

Proof. Let $m>k$. Then from the discussion preceding Theorem 4.2 we know that we may write

$$
A_{m}=A_{k} C_{1} A_{k} C_{2} A_{k} \cdots A_{k} C_{r} A_{k},
$$

where $L\left(C_{i}\right) \leqq L_{k}$ for $i=1,2, \ldots, r$. A straightforward proof by induction yields that each $C_{i}$ has the following property $(*): C_{i}$ is a subblock of $A_{k}[j]$ for some $j \in \mathfrak{A}(k)$ such that if $B_{k}[j]=b_{1} b_{2} \cdots b_{L_{k}}, A_{k}[j]=a_{1} a_{2} \cdots a_{L_{k}}$, and $C_{i}=a_{p} a_{p+1} \cdots a_{p+s}$, then $b_{p}=b_{p+s+1}=1$, where $p+s+1$ is to be reduced modulo $L_{k}$ in case $p+s+1>L_{k}$. It is easy to see that $A_{k+2}$ contains all blocks of the form $A_{k} D A_{k}$, where $D$ is a block having property (*). Therefore each block $A_{k} C_{i} A_{k}$ appears in $A_{k+2}$. Now if $B$ is an $L_{k}$-block which appears in $w$, then $B$ appears in $A_{m}$ for some $m>k$. Writing $A_{m}$ as above, we see that $B$ must appear in one of the blocks $A_{k} C_{i} A_{k}$. Since each block $A_{k} C_{i} A_{k}$ appears in $A_{k+2}, B$ must appear in $A_{k+2}$.

THEOREM 3.3. The topological entropy of $(W, \sigma)$ is zero.

Proof. Lemma 3.2 implies that $\theta\left(L_{k}\right) \leqq L_{k+2}$. Recalling that $m_{k+1}=2 m_{k}^{2}-1$ and $L_{k+1}=\left(4 m_{k}-3\right) L_{k}$, we see that

$$
\theta\left(L_{k}\right) \leqq L_{k+2}=\left(4\left(2 m_{k}^{2}-1\right)-3\right)\left(4 m_{k}-3\right) L_{k} \leqq 32 m_{k}^{3} L_{k},
$$

so

$$
\frac{\log \theta\left(L_{k}\right)}{L_{k}} \leqq \frac{\log 32}{L_{k}}+\frac{3 \log m_{k}}{L_{k}}+\frac{\log L_{k}}{L_{k}}
$$

Since $L_{k} \rightarrow \infty$, it suffices to prove that

$$
\lim _{k \rightarrow \infty} \frac{\log m_{k}}{L_{k}}=0 .
$$

But

$$
\begin{aligned}
\frac{\log m_{k}}{L_{k}} & =\frac{\log \left(2 m_{k-1}^{2}-1\right)}{\left(4 m_{k-1}-3\right) L_{k-1}} \leqq \frac{\log \left(m_{k-1}^{3}\right)}{\left(4 m_{k-1}-3\right) L_{k-1}} \\
& =\frac{3}{4 m_{k-1}-3} \frac{\log m_{k-1}}{L_{k-1}} \leqq \cdots \leqq \frac{3^{k}}{\prod_{i=0}^{k-1}\left(4 m_{i}-3\right)} \frac{\log m_{0}}{L_{0}} \\
& \leqq \frac{3^{k}}{\prod_{i=0}^{k-1}\left(4 m_{0}-3\right)} \frac{\log m_{0}}{L_{0}}=\frac{3^{k}}{9^{k}} \frac{\log 3}{3} \rightarrow 0 .
\end{aligned}
$$

4. Unique ergodicity and failure of measure-theoretic strong mixing. We wish to prove in this section that the flow $(W, \sigma)$ admits a unique normalized invariant Borel measure $\mu$, and that the process [2] $(W, \mathscr{B}, \mu, \sigma)$, where $\mathscr{B}$ is the collection of all Borel subsets of $W$, is not strongly mixing.

In order to prove that $(W, \sigma)$ is uniquely ergodic, it suffices to prove that the defining sequence $w \in W$ is strictly transitive [10]. Specifically, for any two blocks $B$ and $C$ which appear in $w$, let $\nu(B, C)$ denote the number of times that $B$ appears 
in $C$; i.e., $v(B, C)=$ card $\{j: B$ appears at the $j$ th place in $C\}$. Then $w$ is strictly transitive if and only if given a block $B$ which appears in $w$ and $\varepsilon>0$, there is a $k$ such that if $C$ is any $L_{k}$-block which appears in $w$ then

$$
\left|\nu(B, C)-\nu\left(B, A_{k}\right)\right| / L_{k}<\varepsilon .
$$

THEOREM 4.1. $(W, \sigma)$ is uniquely ergodic.

Proof. Let a block $B$ which appears in $w$, say with $L(B)=p$, and $\varepsilon>0$ be given. Since $L_{k-1} / L_{k} \rightarrow 0$ and $m_{k-1} \leqq L_{k-1}$, we may choose $k$ such that $B$ appears in $A_{k-1}, 8\left(L_{k-1} / L_{k}\right)<\varepsilon / 3,8 p\left(m_{k-1} / L_{k}\right)<\varepsilon / 3$, and $6\left(p / L_{k}\right)<\varepsilon / 3$. Let $C$ be any $L_{k}$-block which appears in $w$. We wish to prove that

$$
\left|\nu(B, C)-\nu\left(B, A_{k}\right)\right| / L_{k}<\varepsilon .
$$

To this end we first need to estimate $\nu\left(B, A_{k}\right)$ in terms of $\nu\left(B, A_{k-1}\right)$.

Taking note of the definition of $A_{k}$ in terms of $A_{k-1}$, of the fact that

$$
\left|\nu\left(B, A_{k-1}[j]\right)-\nu\left(B, A_{k-1}\right)\right| \leqq p
$$

for all $j \in \mathfrak{A}(k-1)$, and of the fact that there are $4\left(m_{k-1}-1\right)$ "junctures" of the form $A_{k-1} A_{k-1}[j]$ or $A_{k-1}[j] A_{k-1}$ in the definition of $A_{k}$ (and that the block $B$ can appear no fewer than 0 and no more than $p$ times across any one of these junctures), we conclude that there are $r \in[-1,1]$ and $s \in[0,1]$ such that

$$
\nu\left(B, A_{k}\right)=\left(4 m_{k-1}-3\right) \nu\left(B, A_{k-1}\right)+2 p\left(m_{k-1}-1\right)(r+2 s) .
$$

Now $C$ is an $L_{k}$-block which appears in $w$ and hence, by Lemma 3.2, $C$ appears in $A_{k+2}$. There are now five cases to consider, depending on just which subblock of $A_{k+2}$ the block $C$ happens to be. If $C=A_{k}$ or $C=A_{k}[i]$ for some $i \in \mathfrak{A}(k)$, then the verification of (1) is immediate. Suppose then that $C$ appears in a block $A_{k} A_{k}[i]$ for some $i \in \mathfrak{A}(k)$; the argument in case $C$ appears in a block $A_{k}[i] A_{k}$ will be strictly similar and therefore will not be given here. Now

$$
A_{k} A_{k}[i]=\frac{A_{k}}{A_{k-1} \cdots \underbrace{A_{k-1} A_{k-1}[j] A_{k-1}}_{k-1} \stackrel{A_{k}[i]}{D A_{k-1} A_{k-1}[n] A_{k-1} \cdots}},
$$

where $D$ is a subblock of an allowable permutation of $A_{k-1}$. Counting the minimum and maximum possible numbers of appearances of $A_{k-1}$, of the $A_{k-1}[j]$ 's, and of junctures $A_{k-1} A_{k-1}[j]$ or $A_{k-1}[j] A_{k-1}$ in $C$ yields the estimate

$$
\begin{aligned}
\left(2 m_{k-1}-3\right) \nu\left(B, A_{k-1}\right)+\left(2 m_{k-1}-4\right)\left[\nu\left(B, A_{k-1}\right)-p\right] & \leqq \nu(B, C) \\
& \leqq\left(2 m_{k-1}+1\right) \nu\left(B, A_{k-1}\right)+2 m_{k-1}\left[\nu\left(B, A_{k-1}\right)+p\right]+\left(4 m_{k-1}+1\right) p .
\end{aligned}
$$

With the help of (2) this implies that

$$
\left|\nu(B, C)-\nu\left(B, A_{k}\right)\right| \leqq 3 \nu\left(B, A_{k-1}\right)+p\left(8 m_{k-1}-1\right) \leqq 3 L_{k-1}+8 p m_{k-1},
$$

from which (1) is immediate. The final case to consider is that $C$ appears in a block 
$A_{k} D A_{k}$, where $D$ is a subblock of $A_{k}[r]$ for some $r \in \mathfrak{A}(k)$. A counting procedure similar to the one used in the previous case also establishes (1) for this case. We conclude then that the flow $(W, \sigma)$ is uniquely ergodic.

Thus there is a unique normalized invariant measure $\mu$ on $W$, and $\sigma$ is an ergodic measure-preserving transformation of the measure space $(W, \mathscr{B}, \mu)$. In order to show that the process $(W, \mathscr{B}, \mu, \sigma)$ is not strongly mixing, it is sufficient to display two measurable subsets $A$ and $B$ of $W$ for which

$$
\lim _{n \rightarrow \infty} \mu\left(\sigma^{n} A \cap B\right) \neq \mu(A) \mu(B) .
$$

To this end we will need some quantitative information about the measure $\mu$.

Let $B$ be a block which appears in the sequence $w$ and let

$$
G(B, n)=\{x \in W: B \text { appears at the } n \text {th place in } x\} .
$$

Since $\sigma G(B, n)=G(B, n-1)$ and $\sigma$ preserves the measure $\mu$, we see that $\mu(G(B, n))$ is independent of $n$; we therefore denote $\mu(G(B, n))$ by $\mu^{\prime}(B)$. It is well known that

$$
\mu^{\prime}(B)=\lim _{k \rightarrow \infty} \frac{\nu\left(B, A_{k}\right)}{L_{k}}
$$

For each $k=0,1,2, \ldots$ let $Q_{k}=G\left(A_{k},-\frac{1}{2}\left(L_{k}-1\right)\right)$, and let us estimate

$$
\mu\left(Q_{k}\right)=\mu^{\prime}\left(A_{k}\right)=\lim _{n \rightarrow \infty} \frac{\nu\left(A_{k}, A_{n+1}\right)}{L_{n+1}} .
$$

We have seen earlier that if $m \geqq n$ and $\Lambda^{\prime}=\lambda_{1} \lambda_{2} \cdots \lambda_{s}$, then $\lambda_{j} \geqq k$ implies that the $L_{k}$-subblock of $A_{m}$ appearing at the $j$ th place in $A_{m}$ is $A_{k}$. Let $N(k, m)=$ card $\left\{j\right.$ : the $j$ th entry in $\Lambda_{m}^{\prime}$ is no less than $\left.k\right\}$. Then $N(k, m) \leqq \nu\left(A_{k}, A_{m}\right)$ and, from the definition of $\Lambda_{m}^{\prime}, N(k, n+1)=\left(4 m_{n}-3\right) N(k, n)$ and $N(k, k+1)=2 m_{k}-1$. Thus we have

$$
\begin{aligned}
\nu\left(A_{k}, A_{n+1}\right) / L_{n+1} & \geqq\left(1 / L_{n+1}\right)\left(4 m_{n}-3\right)\left(4 m_{n-1}-3\right) \cdots\left(4 m_{k+1}-3\right)\left(2 m_{k}-1\right) \\
& =\left(2 m_{k}-1\right) / L_{k+1}
\end{aligned}
$$

and hence $\mu\left(Q_{k}\right) \geqq\left(2 m_{k}-1\right) / L_{k+1}$.

THEOREM 4.2. $(W, \mathscr{B}, \mu, \sigma)$ is not strongly mixing.

Proof. For each $k=0,1,2, \ldots$ and $n=0,1,2, \ldots$ let

$$
Q_{k, n}=\left(\sigma^{2 L_{n}} Q_{k}\right) \cap Q_{k} .
$$

We will show that there is a $k$ for which

$$
\liminf _{n \rightarrow \infty} \mu\left(Q_{k, n}\right)>\left(\mu\left(Q_{k}\right)\right)^{2},
$$

and this will show that $(W, \mathscr{B}, \mu, \sigma)$ cannot be strongly mixing.

For each $m, n$, and $k$ with $m>n>k$ let $\nu(m, n, k)$ denote the number of blocks 
of the form $A_{k} D A_{k}$, where $L(D)=2 L_{n}-L_{k}$, which appear in $A_{m}$. Then it is easy to see that

$$
\mu\left(Q_{k, n}\right)=\lim _{m \rightarrow \infty} \frac{\nu(m, n, k)}{L_{m}} .
$$

Let $p \geqq r$ and $\Lambda_{p}=\lambda_{1} \lambda_{2} \cdots \lambda_{s}$; we will say that $A_{r}$ appears explicitly at the $j$ th place in $A_{p}$ if $\lambda_{j} \geqq r$. Now each time $A_{n+1}$ appears in $A_{m}, A_{n}$ appears explicitly $2 m_{n}-1$ times in $A_{n+1}$. Suppose that $A_{n}$ appears explicitly at the $j$ th place in $A_{n+1}$ and that $j<L_{n+1}-L_{n}$; then $A_{n}$ also appears explicitly at the $\left(2 L_{n}+j\right)$ th place in $A_{n+1}$. Therefore, every time that $A_{k}$ appears in such an appearance of $A_{n}$ in $A_{n+1}$, there is another appearance of $A_{k}$ in $A_{n+1} 2 L_{n}$ places along to the right of it. That is to say,

$$
\nu(m, n, k) \geqq N(n+1, m)\left(2 m_{n}-2\right) \nu\left(A_{k}, A_{n}\right) .
$$

It follows then that

and hence

$$
\nu(m, n, k) / L_{m}=\left(2 m_{n+1}-1\right)\left(2 m_{n}-2\right) \nu\left(A_{k}, A_{n}\right) / L_{n+2},
$$

Therefore

$$
\mu\left(Q_{k, n}\right) \geqq\left(2 m_{n+1}-1\right)\left(2 m_{n}-2\right) \nu\left(A_{k}, A_{n}\right) / L_{n+2}
$$

$$
\begin{aligned}
\liminf _{n \rightarrow \infty} \mu\left(Q_{k, n}\right) & \geqq \lim _{n \rightarrow \infty} \inf \frac{\left(2 m_{n+1}-1\right)\left(2 m_{n}-2\right) \nu\left(A_{k}, A_{n}\right)}{L_{n+2}} \\
& =\liminf _{n \rightarrow \infty} \frac{\left(2 m_{n+1}-1\right)\left(2 m_{n}-2\right) \nu\left(A_{k}, A_{n}\right)}{\left(4 m_{n+1}-3\right)\left(4 m_{n}-3\right) L_{n}} \\
& =\frac{1}{4} \lim _{n \rightarrow \infty} \frac{\nu\left(A_{k}, A_{n}\right)}{L_{n}}=\frac{1}{4} \mu\left(Q_{k}\right) .
\end{aligned}
$$

Since $\bigcap_{k=0}^{\infty} Q_{k}=\{w\}$ and $Q_{k+1} \subseteq Q_{k}$, we must have $\lim \mu\left(Q_{k}\right)=0$. Choose $Q_{k}$ such that $\mu\left(Q_{k}\right)<\frac{1}{4}$. Then evidently

$$
\lim _{n \rightarrow \infty} \inf \mu\left(Q_{k, n}\right) \geqq \frac{1}{4} \mu\left(Q_{k}\right)>\left(\mu\left(Q_{k}\right)\right)^{2},
$$

so the process $(W, \mathscr{B}, \mu, \sigma)$ cannot be strongly mixing.

5. Remarks. The construction given above can be varied in many ways to give further examples of strongly mixing minimal sets. For example, in defining $A_{k+1}$ the permutations $A_{k}[j]$ do not have to appear in any particular order, and the $A_{k}$ 's can be repeated more than once between each $A_{k}[i]$ and $A_{k}[j]$; the main requirement is that all allowable permutations should appear and forbidden permutations should be excluded. In general, we may choose a sequence $\left\{m_{k}: k=0,1,2, \ldots\right\}$ of integers such that $m_{k} \geqq 1$ for all $k$ and a block $A_{0}$ with $L\left(A_{0}\right)=L_{0} \equiv 1(\bmod 2)$, and then for each $n \geqq 0$ define

$$
\begin{aligned}
I_{n} & =\left\{-m_{n},-m_{n}+1, \ldots,-1,0,1, \ldots, m_{n}-1, m_{n}\right\}, \\
L_{n+1} & =\left(2 m_{n}+1\right) L_{n}, \quad \text { and } J_{n}=\left\{1,2, \ldots, L_{n}\right\} .
\end{aligned}
$$


If for each $n$ we choose a map $f_{n}: I_{n} \rightarrow J_{n}$ such that $f_{n}(0)=1$ and define

$$
A_{n+1}=A_{n}\left[f_{n}\left(-m_{n}\right)\right] \cdots A_{n}\left[f_{n}(-1)\right] A_{n}\left[f_{n}(0)\right] A_{n}\left[f_{n}(1)\right] \cdots A_{n}\left[f_{n}\left(m_{n}\right)\right]
$$

for all $n \geqq 0$, then the sequence $\left\{A_{n}\right\}$ of expanding central blocks will define an element $x \in S$, which may be called an epicyclic sequence. One-sided sequences can be defined in an analogous manner; in fact, Morse's construction of the Morse-Thue sequence [9] is of this type. As we have seen, many epicyclic sequences will have minimal and strongly mixing orbit closures. Also, homomorphic images of $(W, \sigma)$ under block mappings [5] will be minimal and strongly mixing.

Finally, we conjecture that the process $(W, \mathscr{B}, \mu, \sigma)$ is measure-theoretically weakly mixing.

\section{REFERENCES}

1. R. L. Adler, A. G Konheim and M. H. McAndrew, Topological entropy, Trans. Amer. Math. Soc. 114 (1965), 309-319. MR 30 \#5291.

2. H. Furstenberg, Disjointness in ergodic theory, minimal sets, and a problem in Diophantine approximation, Math. Systems Theory 1 (1967), 1-49. MR 35 \#4369.

3. W. H. Gottschalk and G. A. Hedlund, Topological dynamics, Amer. Math. Soc. Colloq. Publ., vol. 36, Amer. Math. Soc., Providence, R. I., 1955. MR 17, 650.

4. F. Hahn and Y. Katznelson, On the entropy of uniquely ergodic transformations, Trans. Amer. Math. Soc 126 (1967), 335-360. MR 34 \#7772.

5. G. A. Hedlund, "Transformations commuting with the shift," in Topological dynamics, J. Auslander and W. H. Gottschalk (Editors), Benjamin, New York, 1968, pp. 259-289. MR 38 \#2762.

6. K. Jacobs, Systemes dynamiques Riemanniens, (to appear).

7. S. Katutani, Ergodic theory of shift transformations, Proc. Fifth Berkeley Sympos. Math. Statist. Prob. (Berkeley, Calif., 1965/66), vol. II: Contributions to Probability Theory, part 2, Univ. of California Press, Berkeley, 1967, pp. 405-414. MR 37 \#2943.

8. H. B. Keynes and J. B. Robertson, Eigenvalue theorems in topological transformation groups, Trans. Amer. Math. Soc. 139 (1969), 359-370.

9. M. Morse, Recurrent geodesics on a surface of negative curvature, Trans. Amer. Math. Soc. 22 (1921), 84-100.

10. J. C. Oxtoby, Ergodic sets, Bull. Amer. Math. Soc. 58 (1952), 116-136. MR 13, 850.

11. K. E. Petersen, A topologically strongly mixing symbolic minimal set, Notices Amer. Math. Soc. 16 (1969), 180. Abstract \#663-328.

12. - Disjointness and weak mixing of minimal sets, Proc. Amer. Math. Soc. 24 (1970), 278-280.

YALE UNIVERSITY,

New Haven, Connecticut 06520

UNIVERSITY OF NORTH CAROLINA,

Chapel Hill, North Carolina 27514 